\title{
Fernand Dumont (1927-1997)
}

sociologue, Université Laval

(1962)

\section{"L'étude systématique de la société globale canadienne-française"}

\begin{abstract}
Un document produit en version numérique par Jean-Marie Tremblay, bénévole, professeur de sociologie au Cégep de Chicoutimi

Courriel: jmt_sociologue@,videotron.ca

Site web pédagogique : http://www.uqac.ca/jmt-sociologue/
\end{abstract}

Dans le cadre de la collection: "Les classiques des sciences sociales"

Site web: http://www.uqac.ca/Classiques_des_sciences_sociales

Une collection développée en collaboration avec la Bibliothèque

Paul-Émile-Boulet de l'Université du Québec à Chicoutimi

Site web: http://bibliotheque.uqac.uquebec.ca/index.htm 
Cette édition électronique a été réalisée par Jean-Marie Tremblay, bénévole, professeur de sociologie au Cégep de Chicoutimi à partir de :

Fernand Dumont, "L'étude systématique de la société globale canadiennefrançaise". Un article publié dans la revue Recherches sociographiques, vol. 3, no 1-2, janvier-août 1963, pp. 277-294. Québec: PUL.

M. Fernand Dumont (1927-1997), sociologue, enseignait la sociologie à l'Université Laval.

Avec l'autorisation formelle de la directrice de la revue Recherches sociographiques, Mme Andrée Fortin, professeure de sociologie à l'Université Laval, qui nous a demandé de bien mentionner nos sources ainsi que la revue qu'elle dirige.
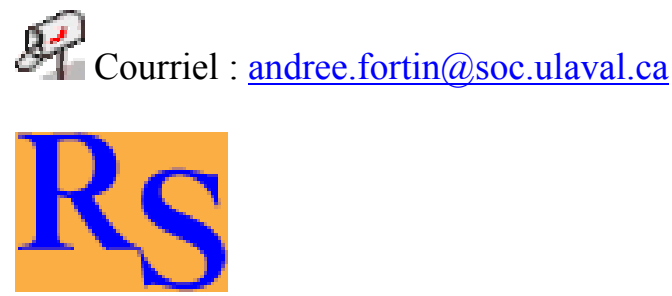

Un gros merci à la revue Recherches sociographiques.

Polices de caractères utilisée :

Pour le texte: Times, 12 points.

Pour les citations : Times 10 points.

Pour les notes de bas de page : Times, 10 points.

Édition électronique réalisée avec le traitement de textes Microsoft Word 2001 pour Macintosh.

Mise en page sur papier format

LETTRE (US letter), 8.5' x 11"')

Édition numérique réalisée le 7 novembre 2004 à Chicoutimi, Ville de Saguenay, province de Québec, Canada. 


\title{
Table des matières
}

Exposé de M. Fernand Dumont

\author{
Introduction
}

I

II

Commentaire de M. Maurice Tremblay, département de science politique, Université Laval. 
Fernand Dumont

\section{"L’étude systématique de la société globale canadienne-française".}

\section{RECHERCHES \\ SOCIOGRAPHIOUES}

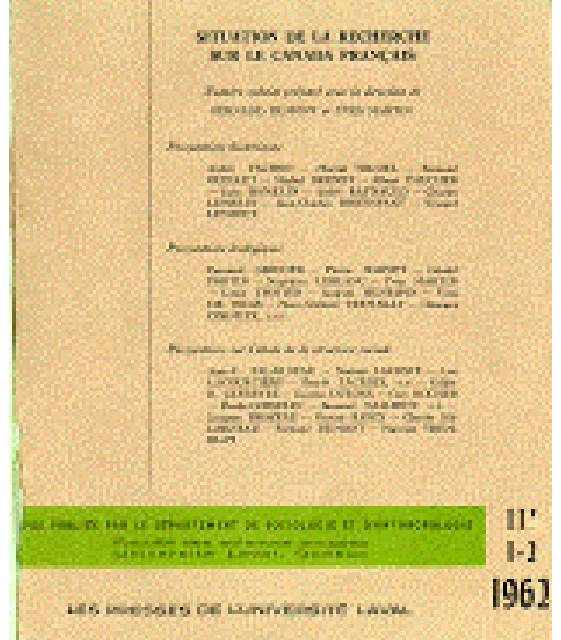

Un article publié dans la revue Recherches sociographiques, vol. 3, no 12, janvier-août 1963, pp. 277-294. Québec: PUL.

M. Fernand Dumont (1927-1997), sociologue, enseignait la sociologie à l'Université Laval. 


\section{"L'étude systématique de la société canadienne-française"}

par Fernand Dumont, 1962

\section{Introduction}

$\underline{\text { Retour à la table des matières }}$

Je tiens à dissiper d'abord une équivoque. Le titre de mon exposé n'implique aucunement que je veuille m'attaquer à une sorte de synthèse des thèmes évoqués au cours de ce colloque. La plupart des auteurs de communications et de commentaires ont bien laissé entendre que l'ère des synthèses-résumés était close pour les chercheurs de cette génération. Parmi toutes les incertitudes dont nous avons fait le bilan au cours de ces journées, voilà un point qui, pour nous tous, est assuré. En parlant de l'étude de la société globale, je ne proposerai donc aucune voie - royale ou tortueuse - pour permettre au sociologue de survoler les lacunes énormes de nos recherches empiriques afin d'en arriver au plus vite à de nouvelles synthèses apaisantes. D'ailleurs, à mon sens (et cela apparaîtra, dans la suite, je l'espère), la notion de société globale n'indique qu'une voie de recherches empiriques parmi bien d'autres.

Cependant, même si ce colloque se déroule sous les auspices de la sociographie, on n'aura aucune difficulté à convenir que le thème que j'ai à traiter appelle des précisions théoriques. La notion de « société globale » apparaît de 
plus en plus comme nécessaire. Nous savons bien que, le plus souvent, la dialectique de la recherche ne va pas de la monographie à la théorie ou inversement. Elle épouse plutôt le schéma : monographie - aire (ou société globale) - théorie. Pourtant, le concept de «société globale » est un des plus confus de la science contemporaine.

Ce n'est évidemment pas le lieu de proposer une discussion purement théorique à ce sujet. Nous ne saurions tout de même éviter de poser tout de suite une question qui s'impose d'emblée : à quels critères nous référer pour délimiter une société globale ? Ce sont de longs développements théoriques qu'il faudrait consacrer à ce problème. Mais nous en sommes dispensés par les impératifs que nous imposent les cadres de ce colloque : notre objet nous était donné au départ. Et, en fait, cela ne gêne pas trop nos idées sur la question. Chaque société globale présente une structure singulière dont le mode d'approche, dans la situation actuelle de la recherche, est à définir à chaque coup. On ne saurait généraliser à propos des sociétés globales comme on le fait, par exemple, en psychologie sociale pour les petits groupes. Ceux-ci s'offrent à la perception comme des ensembles concrets : on est vite renvoyé alors à l'étude des traits généraux de structure. Il n'en est pas ainsi pour les sociétés globales : à première vue, diverses sociétés globales peuvent être délimitées à propos de la même réalité empirique. Nous devons alors fatalement recourir, du moins dans les premières démarches, aux représentations idéologiques où se marque, chez les agents sociaux eux-mêmes, l'appartenance à telle ou telle société globale. On pourra chercher ensuite les mécanismes spécifiques qui soutiennent ces représentations. De sorte que si la notion de société globale apparaît d'abord (nous le notions à l'instant) comme un palier nécessaire de l'observation sociologique, il se pourrait qu'elle corresponde aussi à des éléments concrets et spécifiques des sociétés.

Fidèle à ces remarques comme à l'esprit général du présent colloque, je ne commencerai donc pas par proposer une définition du concept, pour en chercher ensuite l'application à notre milieu. Dans la première partie de mon travail, qui sera consacrée à un bref inventaire, je voudrais plutôt partir avant tout des mécanismes par lesquels la société canadienne-française a tâché de se donner une représentation d'ensemble de ce qu'elle est; l'étude systématique de la société globale nous apparaîtra ainsi en profonde continuité avec son objet. Dans une seconde section de cet essai, je voudrais ensuite proposer de brèves remarques théoriques pour dégager, enfin, quelques propositions de recherche. 
Retour à la table des matières

J'avoue ne pas avoir relevé tous les essais - brefs ou élaborés - où des auteurs ont tenté, depuis deux siècles, de définir le Canadien français. Je me bornerai à mentionner quelques échantillons typiques ordonnés autour d'une classification qui, je l'espère, n'est pas trop simplificatrice. Les tentatives diverses peuvent être rangées, me semble-t-il, en trois courants de pensée $: 1^{\circ}$ les idéologies globales et leurs contestations ; $2^{\circ}$ l'historiographie ; $3^{\circ}$ l'explication de notre société par une référence à la société traditionnelle et à l'urbanisation. Dans notre milieu, ces trois voies de réflexion sont non seulement fort cohérentes pour leur part respective, mais elles se recoupent de façon à former un ensemble lui-même assez organique. J'essaierai, en tout cas, de le montrer.

1. Lorsque le sociologue ou l'anthropologue veut formuler une interprétation d'ensemble d'une société, il se heurte aux grandes idéologies du milieu en question. Avant que nous élaborions la science d'une société, celle-ci s'est déjà donné une vision cohérente de ce qu'elle est. Les idéologies qui nous occupent sont analogues aux rationalisations que doit affronter le psychanalyste. Cette situation comporte une conséquence, entre autres : toute interprétation scientifique d'une société globale sera fatalement en continuité avec les idéologies dominantes du milieu. Parfois, elle en constituera simplement l'explication et la systématisation ; souvent, elle en sera une mise en question chargée plus ou moins de revendications et de jugements de valeur. Oublier cette condition très concrète, bien que gênante, de l'émergence de toute sociologie globale, ce serait nous accorder une objectivité de l'ordre du deus ex machina. On peut donc ainsi parler d'une sorte de polémique sous-jacente à toute interprétation sociologique d'ensemble d'une société globale. ${ }^{1}$

1 On a toujours eu le sentiment plus ou moins confus de cette situation. Pour contourner la difficulté, on a parfois prétendu que toute société ne saurait être convenablement interprétée dans son ensemble que par un étranger. Solution bien précaire, à mon avis : une certaine identification avec l'objet est non seulement inévitable mais nécessaire dans nos sciences; tant que nous ne disposerons pas de techniques pour mesurer ou pour doser cette identification, le recours au «dépaysement» ne saurait être déterminant pour le problème qui nous occupe. D'autant plus que pour se sensibiliser à son objet, le sociologue étranger devrait bien recourir aux grandes représentations locales ou, tout au moins, les confronter avec sa propre explication. 
Cette interpénétration des idéologies dominantes et des sociologies globales est tout particulièrement remarquable au Canada français. Dans une étude rédigée en collaboration avec Guy Rocher, ${ }^{2}$ nous avons essayé de montrer que, chez nous, les idéologies s'étaient intégrées, surtout au cours du du dernier demi-siècle, en un complexe unitaire. Nous avons aussi taché de détecter les grandes antinomies qu'elles tendaient à concilier (le national et le social, la pluralité des allégeances politiques, le religieux et le profane) et les syncrétismes qui les caractérisaient. Devant ce monolithisme, la sociologie canadienne-française ne pouvait éviter la confrontation - qui fut d'ailleurs, conformément à nos remarques de tantôt, le principal moteur de son développement. ${ }^{3}$

Dans un échantillonnage forcement sommaire, il faut ici accorder la première place à l'étude que Maurice Tremblay a publiée dans les Essais sur le Québec contemporain sous le titre : «Orientations de la pensée sociale ». ${ }^{4}$ L'auteur y analyse d'abord les facteurs économiques et idéologiques de ce que l'on a considéré comme la vocation paysanne de la nationalité canadiennefrançaise. Il évoque ensuite brièvement les principaux blocages à notre «pleine réintégration dans la vie économique du continent»: notre système particulier de classes sociales, nos collèges classiques, notre caractère français, notre philosophie catholique de la vie, nos frustrations collectives. Il termine en soulignant brièvement les contradictions du nationalisme économique. Je reconnais une sorte de priorité à ce bref essai parce qu'il est d'une facture remarquable, mais surtout parce qu'il a joué un rôle d'initiateur : on y retrouve, dans une formulation particulièrement nette, le schéma d'explication utilise (souvent avec moins de bonheur) par les auteurs ultérieurs. ${ }^{5}$

2 Fernand DUMONT et Guy ROCHER, «Introduction à une sociologie du Canada français », dans : Le Canada français aujourd'hui et demain (Recherches et débats, cahier no 34), Paris, Fayard, 1961, 13-39.

3 Si, pour donner en quelque sorte un pôle extrême à notre inventaire, nous voulions évoquer une «sociologie » canadienne-française qui ne soit pas autre chose qu'une systématisation de l'idéologie unitaire dont je parlais, c'est probablement l'œuvre de M. Esdras Minville qu'il faudrait retenir comme la plus typique. Retenons cette déclaration de l'auteur : «En fait, ce qui existe, c'est un problème canadien-français présentant un aspect économique comme il présente un aspect social, un aspect politique - aspects que l'on ne saurait s'expliquer complètement si on ne les rattache au fait central » (Esdras MINVILLE, Invitation à l'étude, Montréal, Fides, 1944, 1,26-127). Maurice TREMBLAY a consacré à M Minville une partie notable de son essai : "Réflexions sur le nationalisme » dans : Écrits du Canada français, V, Montréal, 1959. Comme autre exemple, nous aurions pu choisir aussi Notre question nationale du Père Richard ARĖS, s.j., surtout le tome premier (Montréal, Éditions de l'Action nationale, 1944). Mais nous ne pouvons nous attarder : ce genre d'entreprise mérite à peine le nom de «sociologie » puisque, pour reprendre notre analogie avec la psychanalyse, elle représente une sorte de sur-rationalisation. Nous nous attacherons plutôt aux tentatives pour constituer une sociologie par analyse, critique ou remplacement des idéologies dominantes.

4 Dans: Jean-Charles FALARDEAU, éd. Essais sur le Québec contemporain, Québec, Les Presses Universitaires Laval, 1953, 193-208.

5 Je citerai ces quelques lignes qui disent l'essentiel : «Tout changement radical dans les conditions d'existence du groupe qui entraînera un examen critique de la tradition mettra en cause sa structure sociale aussi bien que les valeurs et les normes de son ethos. Pour 
On ne saurait manquer de mentionner, à la suite de l'étude de Maurice Tremblay, l'essai passionne que Pierre-Elliott Trudeau a juxtapose aux monographies portant sur la grève de l'amiante. ${ }^{6}$ On se souvient qu'après un bref rappel des transformations démographiques et économiques au cours de la première moitié de ce siècle, l'auteur y analyse longuement, pour la même période, la pensée nationaliste et ce qu'il appelle «notre doctrine sociale de l'Église » dont il essaie de montrer l'étroite collusion : il décrit ensuite les moyens par lesquels ces doctrines se proposaient de réaliser la restauration économique et sociale du milieu ; enfin, il tâche de déceler les relations entre ce niveau de la pensée et celui des institutions. Trudeau est beaucoup plus polémique (ou plus « engagé », si l'on veut) que Maurice Tremblay. Il déclare : «je ne me ferai pas scrupule de faire ressortir de la pensée nationaliste ces éléments qui encombrent maintenant le présent et nuisent à une action droite et libre $» .{ }^{7}$ Il est souvent question de « nationalisme » sans que jamais on ne nous en propose quelque définition ou tout au moins quelques critères. Surtout, l'auteur pousse si loin l'intention polémique qu'il s'interdit, en définitive, le recours à des explications sociologiques. On ne peut, à la lecture de son essai, que conclure à l'absurdité de la pensée qu'il analyse : mais justement, cela ne saurait satisfaire le sociologue pour qui, par définition, les idéologies ont toujours une signification, c'est-à-dire quelque fondement.

L'essai de Trudeau ne remonte guère au-delà du début du siècle. Bien qu'elle relève d'une tout autre inspiration, une étude de Michel Brunet, qui a eu aussi beaucoup de retentissement, lui fait en quelque sorte pendant. Elle s'intitule : «Trois dominantes de la pensée canadienne-française : il agriculturisme, l'anti-étatisme et le messianisme ». ${ }^{8}$ L'auteur évoque toute l'évolution canadienne à partir des origines. Le constat n'est pas si éloigné du procès de Trudeau : M. Brunet nous parle d' « un faux spiritualisme », d' « angélisme » ... Mais il revient à son explication familière - qu'il oppose d'ailleurs lui-même

autant, la résistance opposée au changement par un système culturel de pensée et par l'organisation sociale qui le supporte sera d'autant plus grande que le nouvel ordre de choses, au lieu d'être le résultat d'une évolution organique du groupe, aura été imposé à celui-ci de l'extérieur. Tel est le cas du groupe canadien-français dans le Québec. L'industrialisation qui transforme son milieu depuis une cinquantaine d'années prend le caractère d'un choc auquel ne l'avaient préparé aucune expérience, aucune évolution interne. Rien d'étonnant que les adaptations exigées par ce bouleversement aient trouvé dans la pensée et dans les institutions traditionnelles une opposition tenace. Cette opposition fut d'autant plus vive que les Canadiens français étaient fortement intégrés dans un cadre social relativement anachronique » (193-194).

6 Pierre-Elliott TRUDEAU, «La Province de Québec, au moment de la grève », dans $L a$ grève de l'amiante, Montréal, les Éditions de Cité Libre, 1956, 1-91.

7 TRUDEAU, ibid., 13.

8 Publiée d'abord dans les Écrits du Canada français, III, Montréal, 1957, 33-117. Réimprimée dans l'ouvrage de l'auteur dans l'ouvrage de l'auteur : La présence anglaise et les Canadiens, Montréal, Beauchemin, 1958, 113-167. 
à celle de Trudeau. ${ }^{9}$ " On ne doit jamais oublier, nous dit-il, que la faiblesse fondamentale de la pensée canadienne-française vient de l'incapacité ou du refus de comprendre le sens véritable de la Conquête de 1760 ». ${ }^{10}$ On ne peut s'empêcher de regretter, chez M. Brunet, cette explication unilatérale. En fixant sans cesse l'attention sur 1760, l'auteur paraît s'interdire de considérer l'importance spécifique de l'histoire subséquente ; tout au moins, celle-ci n'apparaît-elle plus, à la limite, que sous le jour de la fatalité.

Parmi les tentatives pour constituer une sociologie globale de notre société par contestation des idéologies dominantes, je retiendrai un dernier exemple. Il s'agit d'un long article publié naguère dans Cité Libre par Marcel Rioux, sous le titre : «Idéologie et crise de conscience du Canada français. » ${ }^{11}$ À beaucoup d'égards, cette étude de Rioux ajoute des nuances importantes à notre inventaire. Soulignons surtout que l'auteur se donne de longs préalables méthodologiques empruntés aux concepts-clés de l'anthropologie et de la sociologie : 12 il y définit, il est vrai, davantage la «culture » que l'idéologie. La thèse centrale peut être résumée ainsi : «L'idéologie ${ }^{13}$ canadienne-française s'est toujours appuyée sur trois caractères de la culture canadiennefrançaise : minoritaire, catholique et française. C'est à partir de ces caractères, envisagés d'abord dans le concret, mais avec les années de plus en plus schématiquement, que l'idéologie a formulé sa doctrine nationale et qu'elle en est arrivée à contrôler la pensée et la plupart des institutions éducationnelles et intellectuelles du Québec ». ${ }^{14}$ Ici encore, on le voit, la Conquête n'est pas oubliée. Mais la référence essentielle de l'auteur n'est pas là : c'est à partir de la crise de conscience provoquée par l'urbanisation et l'industrialisation que les questions sont, en définitive, posées. ${ }^{15}$

9 Celui-ci « laisse croire, dit-il, que ces hommes de pensée et d'action ont erré parce qu'ils étaient nationalistes. L'explication serait trop simple ! Leur erreur ne vient pas du fait qu'ils ont voulu servir la collectivité canadienne-française. On devrait, au contraire, louer leur sincère désir de travailler au progrès collectif du groupe ethnique dont ils se reconnaissent solidaires. Leur méprise, c'est de n'avoir pas compris la position tragique de la nationalité canadienne-française. Si leurs conceptions économico-sociales ont été fausses ou tronquées, c'est parce qu'ils étaient membres d'une nation démolie et asservie et non parce qu'ils étaient nationalistes. Ils ont eu le tort de croire que la collectivité canadienne-française avait conserve, malgré la Conquête, la pleine maîtrise de ses destinées. C'est ici que l'émotion nationaliste les a trompés » (op. cit., 116, en note).

10 Michel BRUNET, ibid., 117, en note.

11 Marcel Rioux, «Idéologie et crise de conscience du Canada français », Cité Libre, 14, décembre 1956, 1-29.

12 Ibid., 4 et suiv.

13 Remarquer l'emploi du singulier.

14 Marcel Rioux, ibid., 9-10. On mesurera la distance ainsi marquée par un pareil point de départ et la définition de « la culture » proposée par M. le chanoine Groulx: : «le climat physique et spirituel du milieu national, un ensemble de formes ou d'influences qui façonnent un type d'humanité » (Directives, Montréal, Zodiaque, 1937, 142).

15 Voir Marcel Rioux, ibid., 3. Jean-Charles FALARDEAU se place d'un point de vue analogue dans son étude : «Les Canadiens français et leur idéologie », dans : Mason WADE (sous la direction de), La dualité canadienne, Les Presses Universitaires Laval et University of Toronto Press, Québec et Toronto, 1960, 20-38. 
2. L'historiographie d'une société donnée n'est jamais, dans sa structure, sans analogies profondes avec les idéologies dominantes. Cela s'entend facilement. Les hommes recourent à l'histoire pour des raisons semblables à celles qui les poussent è élaborer des idéologies. Dans un milieu social où les schémas de la tradition ne suffisent plus à définir les situations qui les confrontent et à déterminer les conduites à suivre, il faut bien que les hommes édifient consciemment une vision du milieu qui éclaire l'action. Le recours au passé joue alors fatalement un rôle crucial. Les sociétés complexes dont nous héritons prendraient fatalement la figure d'un pesant déterminisme si nous ne pouvions, grâce à l'histoire, en saisir la genèse et le sens et ainsi les restituer à la liberté de nos options. Le lien est ainsi profond et nécessaire entre l'histoire à faire et l'histoire qu'écrivent les historiens. Comme les idéologies, l'historiographie est ainsi une sociologie globale d'un milieu. Plus soucieuse de critique et d'exactitude que les idéologies, elle mérite évidemment d'être privilégiée.

Ces remarques qui valent pour toutes les sociétés s'appliquent tout particulièrement à la notre. Notre historiographie s'est constituée à un moment de crise très aiguë et elle a conservé, par la suite, dans ses schémas d'explication, une très remarquable cohérence. Plus encore peut-être que pour les idéologies dominantes, elle constitue le pôle d'adhésion ou de contestation de toute tentative pour étudier systématiquement notre société globale.

La problématique de notre historiographie s'est fixée très tôt. On en trouve les motivations profondes chez François-Xavier Garneau lorsqu'il déclare, par exemple, dans sa lettre célèbre à Lord Elgin :

«J'ai entrepris ce travail dans le but de rétablir la vérité si souvent défigurée, et de repousser les attaques et les insultes dont mes compatriotes ont été et sont encore journellement l'objet de la part d'hommes qui voudraient les opprimer et les exploiter tout à la fois. J'ai pensé que le meilleur moyen d'y parvenir était d'exposer tout simplement leur histoire. »16

La structure d'ensemble du récit historiographique est fixée dès cette époque. Je citerai a nouveau Garneau :

«Si l'on envisage l'histoire du Canada dans son ensemble, depuis Champlain jusqu'à nos jours, on voit qu'elle se partage en deux grandes phases que divise le

16 François-Xavier GARNEAU, «Lettre à Lord Elgin », dans : Histoire du Canada, éditée par Hector Garneau, 8e édition, Montréal, Éditions de l'Arbre, 1944, 1, 20. - Et se situant, comme par avance, dans le contexte de nos discussions actuelles, il écrit aussi au sujet des Canadiens français : «Leur immobilité apparente tient à leurs habitudes monarchiques et à leur situation spéciale comme race distincte dans l'Amérique du Nord, ayant des intérêts particuliers qui redoutent le contact d'une nationalité étrangère. Ce sont ces deux puissants mobiles qui les ont fait revenir sur leurs pas en 1776, après avoir pour la plupart embrassé un instant la cause américaine ; qui les ont fait courir aux armes en 1812, et qui les ont retenus en 1837 » (ibid., 22). 
passage de cette colonie de la domination française à la domination anglaise, et que caractérisent, la première, les guerres des Canadiens avec les sauvages et les provinces qui forment aujourd'hui les États-Unis ; la seconde, la lutte politique et parlementaire qu'ils soutiennent encore pour leur conservation nationale. » 17

Ce schéma inspirera tous les manuels jusqu'à aujourd'hui et la recherche historique elle-même. Il est en effet extrêmement: curieux de constater que la période de notre histoire qui suit les luttes constitutionnelles n'a pas donné lieu, chez nos historiens, à une structure propre d'explication. On a plutôt, dans certains cas, allongé, « étiré » (pour ainsi dire) la problématique antérieure : c'est pourquoi nous en savons davantage sur les luttes pour les écoles séparées que sur les processus d'urbanisation et d'industrialisation. En somme, les questions essentielles que notre société a posées et formule encore à son passé se sont fixées avant les transformations radicales que ce même milieu a vécues depuis la fin du siècle dernier. La conséquence la plus grave de ce phénomène, c'est que notre historiographie a pris un caractère systématique qui paraît l'éloigner fatalement de nos situations et de nos questions d'aujourd'hui. Un certain pluralisme des explications historiques est inhérent à la conscience historienne quand celle-ci est vraiment le remplacement de la tradition : or, ici, histoire et tradition coexistent en un syncrétisme fâcheux. ${ }^{18}$

Il ne faudrait pas, cependant, exagérer ce monolithisme. On peut discerner des structures internes quelque peu antithétiques. Je signalerai les deux plus importantes à mes yeux.

La première nous renvoie a une dualité d'interprétation de nos relations avec les Anglais et les Canadiens anglais. Dès l'origine de notre historiographie, on peut opposer l'attitude de Michel Bibaud -favorable à l'extrême à la clique du gouverneur de l'époque - et celle de Garneau déjà nationaliste au sens ou nous l'entendons aujourd'hui. Nous avons eu ensuite la dualité Chapais-Groulx : les deux auteurs se sont d'ailleurs opposés directement à propos de la thèse de Chapais sur le caractère « providentiel » de la Conquête anglaise. ${ }^{19}$ Plus récemment on pouvait encore opposer l'École de Montréal et certains historiens comme l'abbé Maheux. Il serait tout à fait fallacieux d'y voir deux traditions rigoureuses : par exemple, on ne saurait, sans être ridicule, faire procéder d'un historien très médiocre et très partial comme Bibaud l'œuvre équitable, mesurée et bien construite de Chapais. Il s'agit plutôt d'une opposition d'attitudes qui renaît, pour ainsi dire, à chaque génération. De plus,

17 GARNEAU, Discours préliminaire. Je cite d'après la première édition, Québec, Imprimerie de N. Aubin, 1845, 19-20.

18 Voir Fernand DUMONT, «De quelques obstacles à la prise de conscience chez les Canadiens français », Cité Libre, 19, 1968, 22-28 ; «La liberté a-t-elle un passé et un avenir au Canada français ? », dans : La liberté, Montréal, Institut canadien des Affaires publiques, 1969, 24-34. Voir aussi Léon DION, « Le nationalisme pessimiste : sa source, sa signification, sa validité », Cité Libre, 18, nov. 1967, 3-19.

19 Voir le parallèle entre Chapais et Groulx tracé naguère par Henri D'ARLES, Nos historiens, Montréal, Bibliothèque de l'Action française, 1921, 193 et suiv. 
l'antinomie n'est pas radicale. Nous l'avons vu : sur le fond de la problématique, sur la sélection des grands événements, la plupart des historiens paraissent profondément d'accord. Pour tous, la société canadienne-française peut être définie globalement sous la figure d'une minorité catholique et française.

Une autre coupure structurelle doit être signalée : celle que représente un groupe d'historiens de l'Institut d'histoire de l'Université de Montréal. Ils sont nationalistes, mais avec des colorations importantes qui les distinguent de leurs prédécesseurs - du chanoine Groulx, en particulier. Pour eux, la Nouvelle-France avait donné lieu à une structure sociale complète dont le dynamisme était représente par une bourgeoisie locale importante. La Conquête est venue bouleverser ces assises de notre nationalité.

Considérée d'ailleurs à un autre niveau, l'école historique dont nous parlons a incontestablement apporté beaucoup à la définition de notre société. Alors qu'elle semble nous emprisonner tragiquement dans un passé assez lointain, les questions qu'elle pose sourdent de nos problèmes les plus actuels ; malgré les apparences, c'est encore le présent (et il en est toujours fatalement ainsi pour l'historien) qui est au départ de l'investigation. En effet, c'est une constatation des faillites des idéologies traditionnelles, de leur peu de réalisme devant nos problèmes d'aujourd'hui, qui différencie, en définitive, les travaux de M. Groulx et ceux de l'école dont nous parlons. On retrouve ainsi un accord profond avec d'autres courants de pensée non nationalistes : la dénonciation des mythes et du patriotisme sentimental n'est pas moins vive chez les uns que chez les autres. Tout en étant, sous bien des aspects, les héritiers de la problématique traditionnelle de notre historiographie, ces jeunes historiens témoignent, a leur manière, de sa faillite. Enfin, il serait tout à fait ridicule de nier la très grande importance de la Conquête dans l'édification de notre société : en ce sens, pour reprendre une expression familière aux sociologues, on peut dire que cette école a construit un modèle partiel parfaitement valable de notre société globale.

3. Poursuivant notre inventaire, nous abordons maintenant le troisième courant de pensée qui a tenté de définir, ici; la société globale : celui qui est axé sur le passage de la société traditionnelle à la société urbanisée et industrialisée. À première vue, nous sommes alors devant un mode de réflexion tout à fait hétéroclite par rapport aux deux précédents : mais la liaison de ce troisième courant avec les deux autres se constate aisément. La contestation des idéologies dominantes et les remaniements de la problématique historiographique procèdent largement, chez nous, de la prise de conscience d'une distance considérable entre la situation présente et les vieilles définitions de la société globale : et ce sont les concepts d' " urbanisation » et d' «industrialisation » qui définissent, le plus souvent, chez les auteurs, cette reconnaissance 
d'une nouvelle situation. ${ }^{20}$ L'étude de l'industrialisation et de l'urbanisation de notre milieu est ainsi, à la fois, la source de l'interrogation sur les représentations idéologiques traditionnelles, en marquant le décrochage subit d'avec les situations d'hier, et la mise en forme des questions à poser au prsent comme au passé de notre société. Pour ce troisième courant de recherches, les aspects polémiques sous-jacents à l'investigation ne sont donc pas négligeables.

La société traditionnelle, qui constitue le premier pôle de référence, a été fortement valorisée par nos idéologies dominantes. On peut dire qu'avant même que les sociologues ou les anthropologues s'y attachent, les idéologies en avaient déjà fait un «type idéal » (ce dernier fermé étant pris à la fois dans le sens de Weber et dans celui plus courant où nous parlons, par exemple, de «l'épouse idéale »!). Il serait donc surprenant, à première vue, que les élaborations proprement sociologiques n'aient pas été contaminées par le contexte idéologique.

Fort heureusement, l'œuvre de celui que l'on peut considérer, par rapport à ce courant d'idées, comme un initiateur, dénote un esprit remarquablement positif. Je veux parler évidemment de Léon Gérin. ${ }^{21}$ Je me bornerai a souligner quelques aspects, que je considère comme importants, de sa sociologie globale.

Dans l'œuvre de Gérin, considérée comme interprétation d'ensemble de notre société, on constate, au départ, une curieuse discontinuité. Le premier sociologue canadien n'a pas négligé l'histoire : plusieurs de ses travaux sur le régime français fournissent encore aujourd'hui à la recherche de stimulantes hypothèses. C'est alors la structure sociale globale qui intéresse Gérin. Et on se rappelle qu'il l'interprète du point de vue d'un phénomène qui lui apparaît comme privilégié : l'entrepreneurship. Mais, sauf pour un article (qui,

20 Marcel Rioux écrit, par exemple : « Aujourd'hui, avec l'urbanisation et l'industrialisation de notre province, une partie toujours grandissante de notre prolétariat sent et comprend que l'idéologie l'a enfermé dans un univers bien mesquin. La crise de conscience du Canada français se reflète chez ces individus; ils voient qu'un profond décalage existe entre l'idéologie et l'existence de tous les jours, entre la théorie et la pratique » («Idéologie et crise de conscience du Canada français », 3). Pour M. Brunet, les nouvelles générations «attendent des chercheurs en sciences sociales les données, les faits et les conclusions dont elles ont un urgent besoin pour éclairer leur action au moment où elles se sentent bouleversées et bousculées par la marche rapide des événements » (la présence anglaise et les Canadiens, 118). Le même auteur oppose « le réalisme de ces nouvelles générations » à l'idéalisme de naguère.

21 Jean-Charles FALARDEAU, «Léon Gérin : une introduction à la lecture de son oeuvre », Recherches sociographiques, 1, 2, avril-juin 1960, 123-160 et "Analyse sociale des communautés rurales », Revue de l'Université Laval, IV, 3, novembre 1949, 210-218 ; Hervé CARRIER, s.j., Le sociologue canadien Léon Gérin, Montréal, Éditions Bellarmin, 1960. L'étude de Falardeau comporte une excellente bibliographie des travaux de Gérin elle nous dispensera de les énumérer ici à notre tour. 
d'ailleurs, porte sur les premières années après la Conquête), ${ }^{22}$ Gérin n'a pas poursuivi, pour les périodes ultérieures de notre histoire, ses études de structures globales. La continuité véritable de son cadre de référence se situe à un autre plan : la famille paysanne, qu'il a étudiée aussi bien à I'origine, en Nouvelle-France, que par observation directe, a la fin du XIXe siècle et au début de ce siècle. Je ne saurais m'empêcher de penser qu'ici, l'École de Le Play - où Gérin, on le sait, avait trouve ses maîtres - lui a joué quelque mauvais tour par la primauté (appuyée surtout sur des postulats idéologiques) qu'elle accordait à la famille et au groupement.

Malgré la valeur des diverses recherches sur notre société traditionnelle, on ne niera pas qu'elles sont bien insuffisantes : ${ }^{23}$ il nous manque, en particulier, trop d'études historiques régionales sur le peuplement. La même constatation s'applique, avec plus de force encore, à l'autre pôle : celui de l'urbanisation et de l'industrialisation. Nous vivons encore largement des constatations faites et des hypothèses formulées par Hugues dans ce classique de nos études sociologiques que constitue French Canada in Transition. ${ }^{24}$ Il faut rappeler aussi, bien entendu, la moisson de faits et de suggestions que constituent les Essais sur Le Québec contemporain. ${ }^{25}$ Mais on admettra que c'est trop peu. La référence à l'urbanisation et à l'industrialisation est devenue si courante dans les propos de nos essayistes et de nos sociologues qu'elle risque de nous conduire à des clichés simplistes plutôt qu'à une problématique complexe. La grande lacune est, ici aussi, du côté des travaux historiques (d'histoire économique, en particulier) qui sont préalables à toute interprétation sociologique un peu complexe de ce genre de phénomènes. ${ }^{26} \mathrm{Ce}$ n'est d'ailleurs pas dans

22 Léon GÉRIN, «L'intérêt sociologique de notre histoire au lendemain de la conquête », Revue trimestrielle canadienne, I, mai 1915, 3-14.

23 Signalons pourtant deux articles de Marcel Rioux où celui-ci, en marge d'une critique des thèses de Philippe Garigue, apporte peut-être à l'étude de notre société traditionnelle les plus précieux éléments depuis Gérin : «Sur le développement socio-culturel du Canada français », Contributions à l'étude des sciences de l'Homme, 4, 1959 ; «Remarques sur les concepts de folk-société et de société paysanne », Anthropologica, 5, 1957, 147-162.

24 Traduit en français par Jean-Charles Falardeau sous le titre : Rencontre de deux Mondes : la crise d'industrialisation du Canada français, Montréal, Parizeau, 1945. Hugues, on le sait, se réfère expressément comme arrière-plan, à Miner et surtout à Gérin. C'est peutêtre Hugues - bien davantage que Gérin lui-même - qui a privilégié, parmi les monographies de Gérin, celle qui porte sur Saint-Justin. Ce passage paraît l'indiquer : «La société rurale du Québec est composée de familles propriétaires de domaines. C'est ainsi que l'a décrite, avec une précision parfaite, le pionnier de nos sociologues, Léon Gérin : «La campagne canadienne-française, écrit-il, se présente comme une simple juxtaposition de familles qui sont à peu près toutes égales ; à peu près toutes engagées dans la culture ; lui presque toutes se suffisent à elles-mêmes, mais dont aucune n'a plus haute ambition que de transmettre intact le bien de famille à quelqu'un de ses enfants, tout en favorisant dans la mesure de ses ressources l'établissement des autres hors du foyer ». La citation renvoie à l'ouvrage de GÉRIN, Le Type économique et social des Canadiens, Montréal, Éditions de l'A.C.F.,1938,84-85.- Or il s'agit justement d'un passage où Gérin parle de Saint-Justin...

25 En particulier, les deux études qu'y a insérées Jean-Charles FALARDEAU ; «The Changing Social Structures » et « Perspectives » (101-122, 239-257).

26 Je me permets ici de souligner la très grande importance des recherches de mon collègue Albert Faucher sur la période pré-confédérative - tout en regrettant qu'elles ne soient pas 
cette direction qu'il faut chercher, à mon sens, la voie d'une sociologie globale de notre milieu.

\section{II}

$\underline{\text { Retour à la table des matières }}$

J'ai souligné, au cours de l'inventaire qui précède, l'unité profonde des trois voies de recherches que nous avons successivement envisagées. Plus directement que je ne l'ai fait jusqu'ici dans mes remarques critiques, je voudrais maintenant formuler quelques propositions au sujet des recherches à entreprendre dans le secteur qui relève de mon exposé. J'aurai alors l'occasion de marquer que rien n'est à renier, pour l'essentiel, dans l'effort déjà accompli ; pourtant, pour indiquer des orientations, on ne saurait éviter de prendre une certaine distance vis-à-vis les démarches poursuivies. Sous forme de réflexions forcement très rapides, il faudra bien dire ce qu'on peut entendre, à un niveau un peu théorique, par le concept de «société globale».

De façon superficielle il est vrai, ce concept semble supposer une sorte de fonctionnalisme intégral - dont Merton a fort bien dénoncé les postulats. Mais les analyses de Merton ne font, à mon sens, que reporter le problème un peu plus loin. Même si nous admettons, sans peine, que tous les éléments d'une société ne sont pas nécessaires a sa structure globale, qu'il y a, au surplus, des fonctions manifestes et des fonctions latentes, la difficulté décisive se pose toujours : comment pourrons-nous identifier, dans un ensemble social un peu complexe, toutes ces dysfonctions, toutes ces fonctions manifestes ou latentes? Nous sommes renvoyés à un inventaire indéfini.

S'il est impossible (et cela paraît incontestable) de montrer, en détail, l'intégration ou la dysfonction de tous les éléments d'une société, le concept serait-il purement gratuit ? Ou tout au plus, n'est-il pas qu'une simple «idée directrice », au sens où Kant parle de la finalité biologique ? Alors - comme Kant l'a aussi montre - une pareille idée a peut-être un sens épistémologique, mais ne saurait prétendre au statut de concept scientifique ; elle orienterait

d'un accès plus facile aux chercheurs. Rappelons, en particulier : (en collaboration avec Maurice Lamontagne), «History of industrial Development», dans : Essais sur le Québec contemporain, 23-37. 
notre pensée mais il nous serait interdit de l'utiliser explicitement dans nos travaux.

Des solutions de remplacement ont été, en fait, envisagées. On a suggéré, par exemple, que l'analyse globale d'une société renvoie à un angle privilégié où la société globale apparaîtrait dans sa spécificité. Et on évoque souvent, en ce sens, les «phénomènes sociaux totaux » de Mauss - que lui-même définissait comme des phénomènes-clefs « où s'expriment à la fois et d'un coup toutes sortes d'institutions ». ${ }^{27}$ Dans une voie toute proche de recherche, on a aussi pensé que certaines crises décisives des sociétés pouvaient permettre d'en atteindre les structures spécifiques. Les analyses de Georges Balandier sur les Fang et les Ba-Kongo sont une fort belle illustration de cette intention. 28

Malgré l'intérêt tout particulier de cette perspective, on ne saurait y voir une solution à notre problème. Car la question essentielle se pose toujours : on ne peut parler de «phénomène social total» ou de « crise concernant la quasitotalité de la société » qu'en se référant, explicitement ou implicitement, à une vision globale de la société en question. Cette vision globale, peut-on en construire une image scientifique explicite?

Il ne reste qu'une voie praticable, à mon sens, pour arriver à une solution. Il n'est pas possible, nous l'avons dit, de supposer que tous les éléments d'une société sont fonctionnels ; le relevé de toutes les dysfonctions, de toutes les fonctions manifestes ou latentes est exclu; la détection des phénomènes ou des crises privilégies où apparaitrait la figure d'ensemble de la société suppose quelques précisions sur les caractéristiques de cet ensemble. Tout cela nous conduit à cette conclusion - qui est aussi une observation assez simple : si une société n'est pas totalement intégrée, on ne peut manquer de constater que chacune possède des mécanismes particuliers et concrets qui travaillent spécifiquement a son intégration globale. La tache de la recherche théorique et empirique, dans le secteur qui nous occupe, se réduirait alors à identifier ces mécanismes, à mesurer leur efficacité et les obstacles qu'ils rencontrent. Entre

27 Marcel MAUSS, Sociologie et anthropologie, Paris, Presses Universitaires de France, 1950,274. [Oeuvre disponible dans Les Classiques des sciences sociales.]

28 L'auteur l'a définie lui-même en ces termes : « Ces crises concernent la quasi-totalité de la société, les institutions comme les groupements et les symboles sociaux. Les désajustements constituent autant d'issues permettant à l'analyse de s'insinuer et de saisir non seulement les phénomènes de contact entre société coloniale et société colonisée, mais encore de mieux comprendre cette dernière dans ses formes traditionnelles en manifestant certaines faiblesses caractéristiques ou certaines structures et représentations collectives irréductibles » (Georges BALANDIER, Sociologie actuelle de l'Afrique noire, Paris, Presses Universitaires de France, 1955, 28). Le même auteur écrit aussi, dans une étude plus récente, à propos du bilaba des Fang: «Ces manifestations totales servent non seulement à "exposer" le système social - en le jouant en quelque sorte sur la place publique - elles ont aussi une efficacité thérapeutique » («phénomènes sociaux totaux et dynamique sociale », Cahiers internationaux de sociologie, XXX, 1961, 34). 
ces éléments sociaux, dont le nombre serait relativement réduit il faudrait établir une analyse fonctionnelle « de second degré », pour ainsi dire.

Sans prétendre établir un inventaire exhaustif de ces mécanismes, je proposerais la liste suivante : les idéologies et l'historiographie (dont je ferais une première catégorie), le système d'éducation scolaire, le pouvoir, les classes sociales. On se rappellera, à ce point, nos remarques initiales. Nous ne prétendons aucunement que cette liste des mécanismes d'intégration convient à toute société globale. Elle nous paraît s'appliquer à la société globale canadiennefrançaise dans le contexte singulier où celle-ci s'offre à l'appréhension du sociologue : c'est justement ce contexte que l'analyse critique de notre première partie visait à définir. Mais, en même temps, dans la ligne d'une théorie générale qui reste à élaborer, cet inventaire nous parait s'ouvrir à la comparaison avec d'autres cas particuliers.

Il faudrait commenter longuement chacun de ces thèmes ; pour faire bref, je me contenterai de proposer quelques remarques sur chacun en les situant dans le contexte des recherches à faire sur la société globale canadiennefrançaise.

1. En premier lieu, j'ai isolé les idéologies et, subsidiairement, l'historiographie. Je crois en avoir suffisamment souligne tantôt la signification du point de vue de la structuration des sociétés globales : ce sont là les représentations que les sociétés élaborent elles-mêmes pour se donner une vue globale de leur existence et de leur avenir. J'ai note aussi le nombre et l'importance des essais récents portant sur les idéologies canadiennes-françaises. En plus des critiques que j'ai déjà faites, une lacune essentielle peut être relevée et qui indique, à mon sens, l'orientation que devraient prendre des investigations prochaines. On n'a pas considéré suffisamment les idéologies comme des phénomènes spécifiques ; le plus souvent, la définition explicite ou implicite des auteurs s'élargit à toute la culture canadienne-française ou encore désigne un ensemble d'attitudes diffuses. Ainsi, Marcel Rioux écrit : « Au Canada français, il y a toujours eu une idéologie; depuis 1760, elle n'a guère varie... » ${ }^{29}$ Beaucoup d'auteurs parlent ainsi de «l'idéologie » canadiennefrançaise. ${ }^{30} \mathrm{M}$. Brunet, dans son étude déjà citée, confond visiblement la notion d'idéologie avec la reconstitution plus ou moins large d'un complexe d'attitudes. C'est ainsi qu'après avoir traité longuement d'« agriculturisme », il écrit en conclusion : "Une nouvelle génération de sociologues, de militants syndicalistes et d'écrivains semble convaincue que notre progrès collectif dépend maintenant de la classe ouvrière ... L'ouvriérisme a remplacé

29 Marcel Rioux, « Idéologie et crise de conscience au Canada français », 9.

30 Rappelons le titre même d'un essai de Jean-Charles FALARDEAU, «Les Canadiens français et leur idéologie », dans : La dualité canadienne, 20-38. 
l'agriculturisme ». ${ }^{31}$ On voit le danger de subjectivité : la perception du chercheur risque de se substituer à la structure propre du phénomène ; les « ismes » sont peut-être autant des auteurs que de la société à étudier. Il n'y a pas la seulement une source de confusion, mais le chercheur s'interdit ainsi de détecter bien des facteurs d'explication. C'est ainsi que M. Trudeau, dans l'étude déjà citée, après avoir composé un système idéologique rigide, ne sait trop comment expliquer l'émergence d'un syndicalisme qu'il reconnaît comme étant dynamique. Le recours au hasard qu'il nous propose explicitement provient sans doute d'un vice initial de méthode, d'une conception trop large de l'idéologie qui ne laisse plus guère de place aux tensions au sein même des idéologies et aux facteurs non idéologiques d'explication.

Les études de nos idéologies devraient donc réduire beaucoup plus strictement leur objet. L'analyse consisterait alors, avant tout, à retrouver des mécanismes de rationalisation : c'est-à-dire les tensions, les antinomies, entre les sources idéologiques diverses, entre les sources idéologiques et les autres éléments du social définis par ailleurs, entre le groupe ou l'institution support de l'idéologie et les autres groupes ou institutions. En somme, après s'être surtout - et un peu prématurément - attachée aux relations entre idéologies et milieux, c'est vers la configuration interne des idéologies que notre attention devrait se tourner. La même réflexion s'applique, à mon sens, à notre tradition historiographique : un travail d'ensemble, mettant en évidence les schèmes d'explication, devrait lui être consacré au plus tôt.

2. Je l'ai suggéré : la restriction du champ d'analyse des idéologies nous conduirait fatalement à nous interroger sur les porteurs de ces idéologies. Celles-ci ayant été définies jusqu'à maintenant de façon trop large, on a été porté à supposer qu'elles étaient partagées soit par les Canadiens français en général, soit par les intellectuels (cette catégorie n'ayant d'ailleurs jamais été, ici, cernée ni historiquement ni sociologiquement avec précision) - ou encore, qu'elles se confondaient avec des attitudes reliées logiquement sans que l'on s'attardât sur leurs titulaires. C'est pourquoi, dans un programme de recherches sur la société globale, le système d'éducation scolaire devrait occuper la première place après les idéologies. Bien entendu, parce que c'est le mécanisme par excellence de transmission des idéologies. Mais ne cédons pas à un fonctionnalisme trop facile : ce qui devrait nous intéresser avant tout, dans le système d'éducation scolaire, du point de vue de la société globale, c'est la différence entre ce que l'on appelle couramment «le développement de l'enfant » et la scolarisation considérée comme processus spécifique. Il est curieux de constater que, dans les ouvrages d'ensemble sur la psychologie de l'enfant, on ne marque pas davantage la coupure radicale qu'y produit l'entrée à l'école. Sans doute parce que cela introduirait une dualité gênante dans les

31 Michel BRUNET, La présence anglaise et les Canadiens, 162. 
sources de l'explication : lorsqu'il commence à fréquenter I'école, l'enfant ne quitte pas seulement l'univers restreint des relations profondément affectives pour entrer dans une société plus anonyme, il est surtout soumis aux normes les plus générales et les plus codifiées de sa société. Les normes qui, dans cette situation exceptionnelle, sont transmises à l'enfant révèlent, de façon très directe, les mécanismes d'intégration de la société globale.

Peu d'investigations ont été menées dans cette perspective. Le groupe de recherches de l'École de pédagogie de l'Université Laval s'y est attaché parmi d'autres thèmes. Une thèse dont j'ai eu la responsabilité cette année nous offre déjà une première coupe dans les données de la question, pour ce qui est de l'enseignement secondaire. ${ }^{32}$ De toute manière, dans le contexte canadienfrançais, une hypothèse décisive me paraît s'imposer : dans notre société, la distance entre les normes vécues dans les comportements quotidiens et celles qui sont transmises par les écoles est tellement grande qu'elle vaut tout particulièrement d'être considérée de près. On se souviendra que, par rapport à une situation de ce genre, Mead et Bateson proposaient de voir la une cause privilégiée d'évolution sociale rapide : ${ }^{33}$ il vaudrait la peine d'essayer de le vérifier dans notre société. ${ }^{34}$

3. En troisième lieu, on n'aura pas de peine a admettre qu'il faut introduire le pouvoir - a condition d'en diversifier largement les mécanismes fonctionnels. Il faudrait en distinguer, pour le moins, trois niveaux : l'État proprement dit, les partis, le pouvoir social. ${ }^{35} \mathrm{Au}$ premier plan, nous disposons déjà d'une bonne esquisse de Maurice Lamontagne ${ }^{36}$ qui, pour être contestable sur certains points, n'en est pas moins extrêmement suggestive. Au niveau des partis, des hypothèses fort plausibles ont déjà été formulées : le caractère abstrait et incolore des partis canadiens qui doivent tenir compte, à la dimension du pays, de problèmes non seulement très divers, mais antinomiques ; la longue emprise du même parti sur le pouvoir, aussi bien au gouvernement fédéral que dans notre province ; l'interférence - abstraite elle aussi - des conflits ethniques et de la politique fédérale ; etc. Cette «abstraction » nous renvoie, ici plus qu'ailleurs peut-être, à l'étude des mécanismes fonctionnels qui relient le pouvoir politique et le pouvoir social. Le « patronage » doit sans

32 Nicole GAGNON, L'idéologie humaniste dans la revue "L'enseignement secondaire », thèse de maîtrise, Département de sociologie et d'anthropologie, Université Laval, 1962.

33 Voir Gregory BATESON et Margaret MEAD, Balinese Character: A Pholographic Analysis, New-York, 1942.

34 Sur l'analyse sociologique d'un système d'éducation, en particulier du nôtre, je me permets de renvoyer à mon étude : «Scolarisation et socialisation : pour un modèle général d'analyse en sociologie de l'éducation », à paraître dans Contributions à l'étude des sciences de l'homme (Montréal).

35 Voir mon article à paraître dans un prochain numéro de Recherches sociographiques, De la sociologie politique en général à celle du Canada français. »

36 Maurice LAMONTAGNE, Le fédéralisme canadien -évolution et problèmes, Québec, Les Presses Universitaires Laval, 1954. 
doute, en ce sens, retenir notre attention : si nous pouvions déterminer en quoi il répond aux besoins locaux et régionaux qui, dans notre milieu, ne reçoivent pas une réponse d'autre manière, nous saurions du même coup beaucoup de choses sur notre société. Mais, la liaison précise du pouvoir politique et du pouvoir social resterait encore à chercher, me semble-t-il, dans une direction que paraît indiquer clairement notre histoire : je veux parler de la politisation traditionnelle, dans notre milieu, des voies d'accès au monde des élites. Edmond de Nevers a naguère (1896) donné sur ce point des observations qui sont encore éclairantes. Mais des élites nouvelles apparaissent dans notre société : dans le milieu syndical et dans le mouvement coopératif, en particulier. Ces leaders élaborent de nouveaux éléments idéologiques encore mal intégrés, mais qui révèlent sans doute les colorations diversifiées des attitudes des Canadiens français. Ce phénomène donne lieu à des spéculations sociologiques et politiques bien générales ; une sociologie globale de notre société devrait, il me semble, s'y attacher minutieusement.

4. Cette recherche sur les élites nous amène naturellement aux classes sociales. Sur ce point particulièrement, la recherche est, ici, dans l'enfance. Beaucoup de préjugés, hérités des classes moyennes - comme aux États-Unis, - nous empêchent de poser facilement le problème. Et, par surcroît, nous sommes souvent obsédés encore par la nostalgie d'une activité économique qui serait aux mains des Canadiens français. Pourtant, c'est bien par rapport aux classes sociales que les questions que nous nous posons au sujet des idéologies dominantes prennent toute leur signification. Toutes nos discussions chez les sociologues comme chez les historiens -sur le «nationalisme », « l'agriculturisme » et autres denrées de ce genre devraient nous amener à une question décisive : quelles strates sociales ont eu intérêt à fabriquer de pareilles productions intellectuelles ? Je ne parle évidemment pas seulement d'intérêts économiques. C'est pourquoi j'élargirais ma question : aux difficultés et aux angoisses de quels groupements ont répondu nos productions idéologiques? Nous retrouvons ainsi le noyau du programme de recherche que j'ai essayé d'esquisser très superficiellement. C'est pourquoi, à mon avis, les recherches sur le leadership et les classes sociales délimitent, particulièrement dans notre milieu, la problématique d'une étude de la société globale.

\section{Fernand DUMONT}

Département de sociologie et d'anthropologie, Université Laval. 


\title{
Commentaire
}

\author{
Maurice TREMBLAY \\ Département de science politique, \\ Université Laval.
}

$\underline{\text { Retour à la table des matières }}$

J'avoue que je suis un peu gêné d'avoir à amorcer la discussion Sur la communication de monsieur Dumont. D'abord, parce que la place qu'il a accordée, dans son inventaire, à mon étude des Essais sur le Québec contemporain me donne la troublante impression d'être réduit à la catégorie inerte des objets qu'on analyse et qu'on dissèque et ou l'on se sent un peu comme paralyse. Et le fait que cette place soit privilégiée, au-delà même du mérite facile de mon essai d'être l'un des premiers du genre, ne fait qu'ajouter à ma gêne.

Pour ma part, je vois dans cette espèce de traitement de faveur qu'il m'accorde une bonne illustration de la difficulté qu'a tout sociologue d'atteindre à la parfaite objectivité, biaisé qu'il peut être par ses sentiments personnels relatifs a son objet d'étude et, entre autres, par ses sentiments d'amitié. Et cette illustration de l'influence insidieuse de la subjectivité est d'autant plus probable que l'auteur de la communication m'a avoué avoir eu des scrupules à donner une appréciation objective de mon essai, alors qu'il me savait invité à faire le premier mes commentaires et qu'il ne voulait ni m'influencer ni même avoir l'air de m'influencer en sa faveur.

Je ne doute pas de la sincérité de ces propos ; mais vous reconnaîtrez avec moi qu'ils n'avaient qui puisse dissiper mon malaise ni atténuer le conditionnement dont M. Dumont se faisait scrupule. Mais comme je ne pouvais plus me récuser, il ne me restait, en préparant mes remarques, qu'à tâcher de dominer ma gêne et à rechercher à mon tour l'objectivité, en essayant de résister à la tentation de la condescendance reconnaissante. 
La chose m'est d'ailleurs facilitée, car je crois m'être rendu compte qu'en plus du préjugé favorable inconscient un défaut de méthode expliquait la place avantageuse que je partageais avec Marcel Rioux dans l'analyse comparative des quatre essais choisis par l'auteur comme échantillons, dans la catégorie des études portant sur les idéologies traditionnelles.

Si nous avons trouvé grâce devant l'auteur ne serait-ce pas parce que nos études étaient les deux seules de l'échantillon qui aient eu des prétentions sociologiques et qui aient eu quelque chance de répondre aux exigences «d'une interprétation scientifique » et de constituer une contribution à «la sociologie globale » de notre milieu ?

On peut être justifié de considérer que l'essai de Pierre-Elliott Trudeau constitue une étrange introduction aux autres études contenues dans La grève de l'amiante, mais peut-on légitimement lui appliquer rigoureusement les canons de la sociologie alors que l'auteur se situe d'emblée au plan normatif de la contestation des idéologies traditionnelles, considérées non pas comme objets à analyser spéculativement, mais uniquement dans la mesure ou, comme le dit Trudeau, et comme le note d'ailleurs l'auteur, certains de leurs éléments « encombrent maintenant le présent et nuisent à une action droite et libre ».

Sans doute, ce genre de contestation ne dispense-t-il pas de toute analyse objective et peut-être que Pierre-Elliott Trudeau ne s'est pas appliqué assez systématiquement a faire ressortir tous les facteurs qui ont pu conditionner nos « définisseurs de situation » traditionnels et qui pouvaient même leur donner en partie raison à l'époque; en sorte qu'il a pu les faire paraître moins lucides qu'ils ne l'avaient été en réalité. Mais peut-être importe-t-il de rappeler que l'explication la plus exhaustive n'équivaut jamais a une justification et que le but de Trudeau, dans son essai, était précisément de montrer que nos maitres à penser s'étaient largement trompes dans l'évaluation de la situation qu'ils prétendaient définir ; ce qui est parfaitement légitime, mais ce qui implique nécessairement, comme tout jugement de valeur, un engagement de l'auteur beaucoup plus explicite qu'une simple analyse sociologique qui, elle, peut se permettre d'être beaucoup plus détachée et plus sereine.

Dans la mesure où l'essai de Michel Brunet se présente lui aussi comme une contestation de certaines idéologies traditionnelles, je considère qu'il est normal qu'il prenne à son tour l'allure d'un procès et qu'il devient alors difficile de le comparer, sans transgression de genres, à des essais qui se réclament exclusivement de la sociologie et de ses méthodes. Ce qui ne m'empêche pas, bien sur, de reconnaître le bien-fonde des critiques formulées par l'auteur au su) et des explications ou plutôt de l'explication sociologique sur laquelle Michel Brunet appuie ses jugements de valeur. 
Il existe par ailleurs un essai proprement sociologique concernant nos idéologies qui se prêtait de soi à la comparaison dans ce contexte et qui l'aurait supportée très avantageusement. Il s'agit, vous l'avez facilement devine, de l'essai en tous points remarquable publié par Fernand Dumont et Guy Rocher sous le titre d' "Introduction à une sociologie du Canada français » dans le numéro de la collection Recherches et débats consacré au Canada français. L'auteur de la communication y a fait une allusion discrète, mais il pouvait difficilement en faire la recension qui s'imposait. Il me revient donc de combler cette lacune, ce qui me permettra de payer ma dette envers Fernand Dumont avec la meilleure conscience du monde.

L'intérêt de cet essai est d'abord méthodologique en ce qu'il suit de très près l'approche féconde que propose Fernand Dumont a la fin de sa communication pour l'étude des sociétés globales par l'analyse fonctionnelle de leurs idéologies.

Quant au contenu, l'essai complète très heureusement sous plusieurs aspects les études antérieures. D'abord en ce qu'il étudie plus systématiquement qu'on ne l'avait jamais fait la genèse de l'ideologie dominante dans la première partie du XXe siècle, en montrant tout particulièrement, par des références à ce grand méconnu qu'est notre XIXe siècle, que son monolithisme ne devait pas être projeté trop en arrière dans l'histoire. Mais la principale contribution des auteurs est d'avoir analysé l'éclatement de ce bloc idéologique qui est en train de s'opérer sous le pouvoir des forces nouvelles par la dissociation du social et du national, du religieux et du profane et même, à l'intérieur de l'Église, par l'affranchissement du laïcat du contrôle clérical.

Je clos là mes remarques laissant à d'autres le soin de les compléter, et en particulier aux historiens celui de commenter les considérations de l'auteur sur l'évolution de notre historiographie.

Je me contenterai en conclusion de souhaiter, dans le prolongement des vœux de Fernand Dumont, que celui qui dans dix ans aurait à recenser les contributions à une analyse de notre société globale, puisse faire état d'études substantielles et non plus seulement d'une courte série d'essais.

Maurice TREMBLAY

Département de science politique, Université Laval.

Fin du texte 